\title{
Holstein Friesian dairy cattle edited for diluted coat color as a potential adaptation to climate change
}

\author{
G. Laible ${ }^{1,2,3^{*}}$, S.-A. Cole ${ }^{1}$, B. Brophy ${ }^{1}$, J. Wei ${ }^{1}$, S. Leath', S. Jivanji ${ }^{4}$, M. D. Littlejohnn ${ }^{4,5}$ and D. N. Wells ${ }^{1}$
}

\begin{abstract}
Background: High-producing Holstein Friesian dairy cattle have a characteristic black and white coat, often with large proportions of black. Compared to a light coat color, black absorbs more solar radiation which is a contributing factor to heat stress in cattle. To better adapt dairy cattle to rapidly warming climates, we aimed to lighten their coat color by genome editing.

Results: Using gRNA/Cas9-mediated editing, we introduced a three bp deletion in the pre-melanosomal protein 17 gene (PMEL) proposed as causative variant for the semi-dominant color dilution phenotype observed in Galloway and Highland cattle. Calves generated from cells with homozygous edits revealed a strong color dilution effect. Instead of the characteristic black and white markings of control calves generated from unedited cells, the edited calves displayed a novel grey and white coat pattern.

Conclusion: This, for the first time, verified the causative nature of the PMEL mutation for diluting the black coat color in cattle. Although only one of the calves was healthy at birth and later succumbed to a naval infection, the study showed the feasibility of generating such edited animals with the possibility to dissect the effects of the introgressed edit and other interfering allelic variants that might exist in individual cattle and accurately determine the impact of only the three bp change.
\end{abstract}

Keywords: Cas9, Genome editing, Homology-directed repair, PMEL, P.Leu18del, Coat color, Cattle, Somatic cell nuclear transfer, White spotting, Signal peptide

\section{Background}

The present trend of increasing global temperatures is rapidly changing the environment and conditions under which dairy animals are grazing. Hence, dairy cattle are no longer well adapted to the predicted realities of more frequent and prolonged periods of hotter summer temperatures [1-4]. This poses significant challenges for their welfare and negatively impacts on their productivity [5-7]. Already, New Zealand dairy cows become heat stressed for close to $20 \%$ of lactation days in major dairy

\footnotetext{
*Correspondence: goetz.laible@agresearch.co.nz

${ }^{1}$ AgResearch, Ruakura Research Centre, Hamilton 3240, New Zealand

Full list of author information is available at the end of the article
}

regions in New Zealand [8]. This is particularly relevant for animals with black hair, a common characteristic of Holstein Friesian dairy cattle, which absorb twice as much solar radiation as white hair [9]. Hence, it exposes black animals to enhanced radiative heat gain which contributes to heat stress. In hot weather, this results in a reduced ability of primarily black dairy cows to regulate body temperature and maintain milk production levels [10] while also negatively impacting on their reproductive performance [11]. Lightening of the coat color should help to reduce these impacts and provide a first step to better adapted dairy cattle. In different species, $P M E L$ mutations were shown to be associated with color dilution effects [12-17]. A deletion of a leucine codon in the 
signal peptide (p.Leu18del) of PMEL segregates in Highland, Galloway, and Tuli cattle, and has been proposed as a causative mutation for coat color effects $[18,19]$. Animals heterozygous for the p.Leu18del mutation display a faded version of the wt black or red coat color, referred to as dun or yellow, respectively. Homozygotes present an even stronger dilution effect with a white to off-white coat, also called silver dun (black wt) or white (red wt) [18]. While the role of this variant has not been functionally confirmed, its proposed coat color impacts make it an excellent candidate for introgression into Holstein Friesian dairy cattle to reduce radiative heat gain and improve overall heat tolerance. Although the introgression of the p.Leu18del mutation would be possible with a conventional crossbreeding strategy, such an approach would not enable immediate functional validation of the p.Leu18del variant since many other variants on the same chromosome are co-inherited when crossbreeding. In addition, it would require back crossing over many years to catch up to the genetic merit of contemporaneous animals, which renders this process unsuitably slow for a timely adaptation. By contrast, genome editing can prove the causative relationship between a specific mutation and impact on phenotype, and provide the scope for rapid introgression - essentially within a single generation $[20,21]$.

In this study, we report the gRNA/Cas9-mediated introgression of the naturally occurring mutation p.Leu18del in the PMEL gene known from Galloway and Highland cattle, into Holstein Friesian cattle. Calves homozygous for the $P M E L$ mutation displayed a distinct color dilution to a silvery grey of the otherwise black coat markings in the control calves. The white areas remained unaffected. This novel grey and white coat color phenotype demonstrates the causative role of the $P M E L$ mutation in coat color dilution. In addition, our study shows that the introgression of naturally occurring sequence variants by genome editing has potential as a new approach to rapidly improve and adapt livestock to changing environmental conditions.

\section{Results gRNA/Cas9-mediated editing of the p.Leu18del PMEL mutation}

The three bp deletion $P M E L$ variant, resulting in the deletion of leucine 18 in the signal peptide of PMEL, is known from Highland cattle where it is associated with a semi-dominant color dilution phenotype [18].

To introgress this naturally occurring sequence variant into Holstein Friesian cattle we first designed three different gRNAs targeting the $P M E L$ gene sequence near the mutation site. The corresponding gRNA/Cas9 editors were then evaluated by digital droplet PCR (ddPCR) for target-specific cleavage activity and efficiency for template-directed repair. The analysis of primary bovine embryonic fibroblast cells (BEF2) individually transfected with the gRNA/Cas9 editors 122_F, 129_F and 130_F showed that all three editors could generate indel mutations at the target site following NHEJ repair (Table 1). The best mutation rate was attained using gRNA/Cas9 editor 129_F with $35 \%$ of sequences containing indel mutations. To measure homology-directed repair (HDR) efficiency we co-transfected BEF2 cells with the gRNA/ Cas9 editors and a 127 bp ss oligonucleotide specifying the three bp deletion of the p.Leu18del PMEL mutation (Fig. 1). This confirmed that the highest HDR activities were associated with gRNA/Cas9 editors 122_F and 129_F and resulted in 5.7 and 6.0\% of HDR events, respectively in transfected BEF 2 cells.

\section{Isolation of precisely edited cell clones}

Based on our initial testing, we selected gRNA 129_F for the gRNA/Cas9-mediated editing of BEF2 cells for the isolation of edited cell clones. Following co-transfection of the PMEL-specific editors and HDR template, we picked 128 individual mitotic cells into 96 well plates for clonal expansion. Of those, 96 expanded into cell clones that were characterised (Table 2). The isolated cell clones were first screened by mutation-specific PCR, that was designed to only amplify the three bp deletion allele. This initial screening was done at low stringency to minimize the risk of failing to detect some correctly edited cell clones. It identified 30 candidate clones for the intended HDR edit. These candidates were then more stringently screened by ddPCR using a hydrolysation probe specific for the three bp HDR mutation. This revealed a total of seven cell clones that had at least one HDR allele, and in combination with sequencing and TIDE analysis, three cell clones were confirmed for one HDR and one wt allele (monoallelic) and four cell clones were biallelically edited with two HDR alleles (Fig. 2).

\section{Generation of edited calves}

To determine the phenotypic impact of the PMEL mutation we used somatic cell nuclear transfer (SCNT) to generate calves with donor cells from the biallelic cell clone $\mathrm{CC} 14$ and the parental wt cell line BEF2. Following

Table 1 Target-specific editing activity of three PMEL-specific gRNA/Cas9 editors

\begin{tabular}{lll}
\hline gRNA/Cas9 editor & \% NHEJ & \% HDR \\
\hline 122 F & 11 & 5.7 \\
$129 \_F$ & 35 & 6.0 \\
$130 \_F^{\text {a }}$ & 7 & 3.8 \\
\hline
\end{tabular}

${ }^{a}$ based on a single transfection 


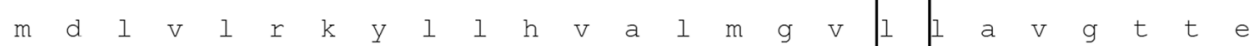 ...ATGGATCTGGTGCTGAGAAAATACCTTCTCCATGTGGCTCTGATGGGTGTT CTTCTGGCTGTAGGGACCACAGAAG... $\ldots|||||||||||||||||||||||||||||||||||||||||||||||||||||||||||||||||||||||||||||||| \mid \ldots$ ...tACCTAGACCACGACTCTTTTATGGAAGAGGTACACCGAGACTACCCACAA GAAGACCGACATCCCTGGTGTCTTC... \\ CTTTTATGGAAGAGGTACACCGAGACTACCCACAA---GACCGACATCCCTGGTGTCTTC+70nts

Fig. 1 Schematic overview of the PMEL target region. Shown is the DNA sequence for the relevant region of the PMEL gene with the binding site for editor 129_F (bold, underlined), PAM sequence (grey highlight) and the location of the three bp deletion (box). Below is the aligned sequence of the ss HDR template (ssODN) specifying a three bp deletion (dashes). The corresponding amino acid sequence for PMEL is given in single letter code above the DNA sequence with lower case indicating amino acids of the predicted signal peptide according to the UniProt annotation for bovine PMEL (entry Q06154)

Table 2 Summary of cell clone isolation

\begin{tabular}{lll}
\hline Isolation/Characterisation Step & $\begin{array}{l}\text { No. of Cell } \\
\text { Clones }\end{array}$ & Efficiency \\
\hline Cell clones picked & 128 & $100 \%$ \\
Expanded cell clones & 96 & $75 \%(96 / 128)$ \\
Mutation specific PCR + ve & 30 & $31 \%(30 / 96)$ \\
ddPCR HDR + ve, total & 7 & $7 \%(13 / 96)$ \\
ddPCR HDR + ve, monoallelic & 3 & $3 \%(3 / 96)$ \\
ddPCR HDR + ve, biallelic & 4 & $4 \%(4 / 96)$ \\
\hline
\end{tabular}

transfer of $22(\mathrm{CC} 14)$ and $14(\mathrm{BEF} 2)$ reconstructed embryos a total of seven pregnancies were detected at day 37 of gestation (Table 3). For each genotype, one pregnancy failed, resulting in the birth of two PMEL mutant calves and three control calves. The health of one of the PMEL calves was compromised at birth due to complication from a hydrops pregnancy. Although the second edited calf was healthy at birth, it died at the age of 4 weeks due to an undetected naval infection. All three control calves were also healthy at birth and developed normally.

\section{Genotype and phenotype characterisation}

The genotype of the calves was confirmed by sequencing of the target region. Genomic DNA isolated from the calves revealed uniform sequences indicating the presence of two identical alleles. Both PMEL mutant calves were of the three bp deletion genotype whereas the genotype of the control calves was wt (Fig. S1). The known interaction of the PMEL mutation with the melanocortin 1 receptor gene $(M C 1 R)$ prompted us to also confirm the MC1R genotype. Animals with the dominant $E^{D}$ allele can only produce the black pigment eumelanin. As expected for black Holstein Friesian cattle as the chosen background genetics, the edited calves were homozygous for the $\mathrm{E}^{\mathrm{D}}$ allele of $M C 1 R$ (Fig. S2).

Because the gRNA/Cas9 editors were delivered as plasmids, the genomic DNA of the calves was assessed for potential vector integration by PCR. PCR amplification of a $472 \mathrm{bp}$ vector-specific fragment from the calves' genomic DNA failed for both PMEL mutant calves and the three control calves and only the positive control produced an amplification product (Fig. S3A, S4A). By contrast, amplification of a genomic fragment from an endogenous bovine gene was successful for all calves (Fig. S3B, S4B). This result suggests that the edited calves were free of unwanted plasmid vector integrations.

To test specificity of the gRNA-Cas9 editor 129_F for the PMEL target site, the top three potential off-target sites, as identified by CRISPOR, were PCR amplified from the edited calves and sequenced. This confirmed that no mutations were introduced at these off-target sites (Fig. S5) and adjacent sequences interrogated by the amplified PCR fragments.

Both PMEL mutant calves, homozygous for the edited three bp deletion, showed a diluted coat color phenotype (Fig. 3). Instead of the typical black and white coat color pattern of the Holstein Friesian cattle breed displayed by the wt control calves, the dark coat markings of the two mutant calves were no longer black but diluted into a much lighter shade of color. This resulted in a striking coat color phenotype of grey and white. Commonly, the color patterns of cloned cattle are not identical but remain very similar, which was the case for the three control calves. By contrast, the pattern of the PMEL mutant calves diverged beyond similarity of the observed pattern of the wt control clones. The markings on the face of the calves serve as an example to best illustrate this exaggerated depigmentation phenotype. Whereas the wt calves possessed a very characteristic black face with a white diamond shape on the forehead, both PMEL mutant calves had a white face. In addition, the total white areas on the coat appeared to be increased in the PMEL mutant calves compared to the wt control calves (Fig. 3). Given that major-effect QTL for white spotting have recently been reported in NZ dairy animals [22], we characterised the status of these loci in our foundation cell lines to provide context to the apparent impacts of p.Leu18del on 


\section{CC24 (monoallelic)

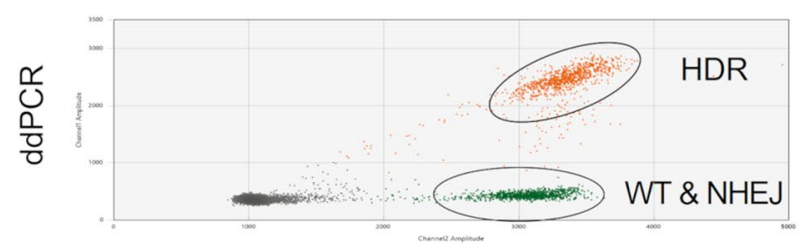 \\ $R^{2}=0.97$ \\ AATACCTTCTCCATGTGGCTCTGATGGGTGTTCTTCTG GCTGTAGG GACCACAGAAG GTGAGTGTGG GATGI}

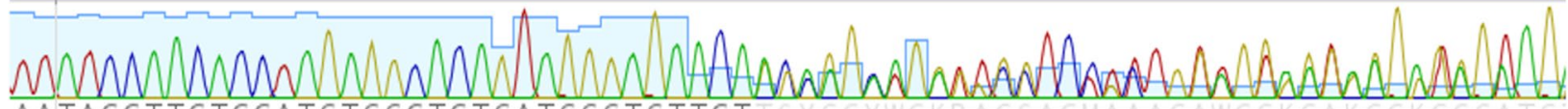
AATACCTTCTCCATGTGGCTCTGATGGGTGTTCT

\section{CC14 (biallelic)}
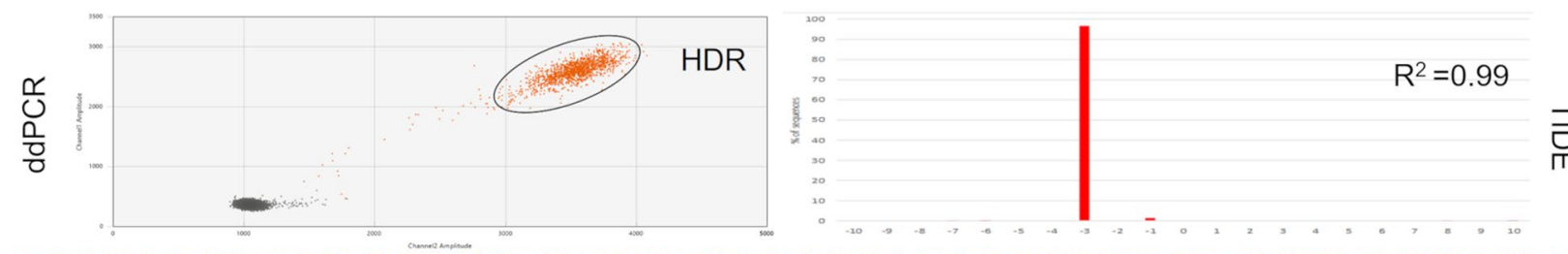

AATAC CTTCTCCATGTGGCTCTGATGGGTGTTCTTCTG GCTGTAG G GACCACAGAAGGTGAGTGTGG GATG

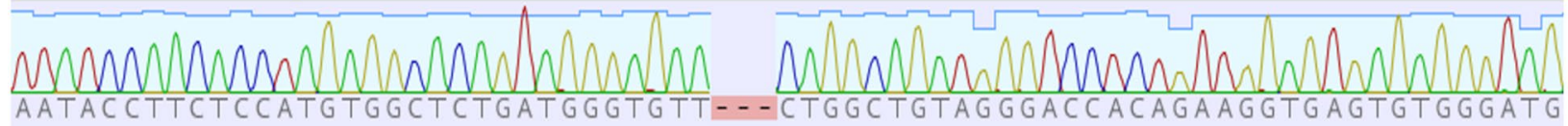

Fig. 2 Monoallelic and biallelically edited cell clones. Examples of cell clones with a monoallelic (CC24) and biallelic (CC14) PMEL HDR edit. Shown are the results for their characterisation by ddPCR, sequencing and TIDE analyses. HDR: pool of droplets recognised by the HDR probe; WT \& NHEJ: pool of droplets not recognized by the HDR probe indicating the presence of a wt or NHEJ allele; $R^{2}$ : coefficient of determination

spotting. This analysis showed edited and control animals were homozygous for two of the three 'white increasing' alleles reported by Jivanji and co-workers [22], suggesting the genetic background of our animals was near 'maximally-spotted' as determined by these other major coatcolor genes (Table S2).

To quantify the color dilution effect caused by the PMEL mutation, colorimetric measurements of hair and underlying skin color were taken from the three control calves and the viable PMEL calf. The characteristic measured was the lightness of the color expressed as $\mathrm{L}^{*}$ value according to the CIELab color scheme which defines the lightness of white as 100 and black as zero. The color shade of the white hair of the PMEL calf $\left(\mathrm{L}^{*}=81.6\right)$ was indistinguishable from the white hair of the three wt control calves determined as 80.8, 83.5 and 81.3 (Fig. 4). This was in stark contrast to the shade of the dark hair, with a strong increase of the lightness for the PMEL calf $\left(\mathrm{L}^{*}=53.8\right)$ compared to the three wt control calves $\left(L^{*}=16.8,20.5\right.$ and $\left.16.5 ; P<0.0001\right)$. To assess any associated changes in the pigmentation of the underlying skin, lightness was measured for the skin below white and

Table 3 Summary of nuclear transfer results

\begin{tabular}{llllll}
\hline Cell clone & Genotype & Embryos transferred & $\begin{array}{l}\text { Pregnancies at D37 of } \\
\text { gestation (\%) }\end{array}$ & $\begin{array}{l}\text { Development to term } \\
(\%)^{\mathbf{a}}\end{array}$ & $\begin{array}{l}\text { Development } \\
\text { to weaning } \\
(\%)^{\mathbf{a}}\end{array}$ \\
\hline CC14 & 3 bp deletion & 22 & $3(14)$ & $2(9)$ & $0(0)$ \\
BEF2 & wt & 14 & $4(29)$ & $3(21)$ & $3(21)$ \\
\hline
\end{tabular}

\footnotetext{
a per total number of transferred embryos
} 

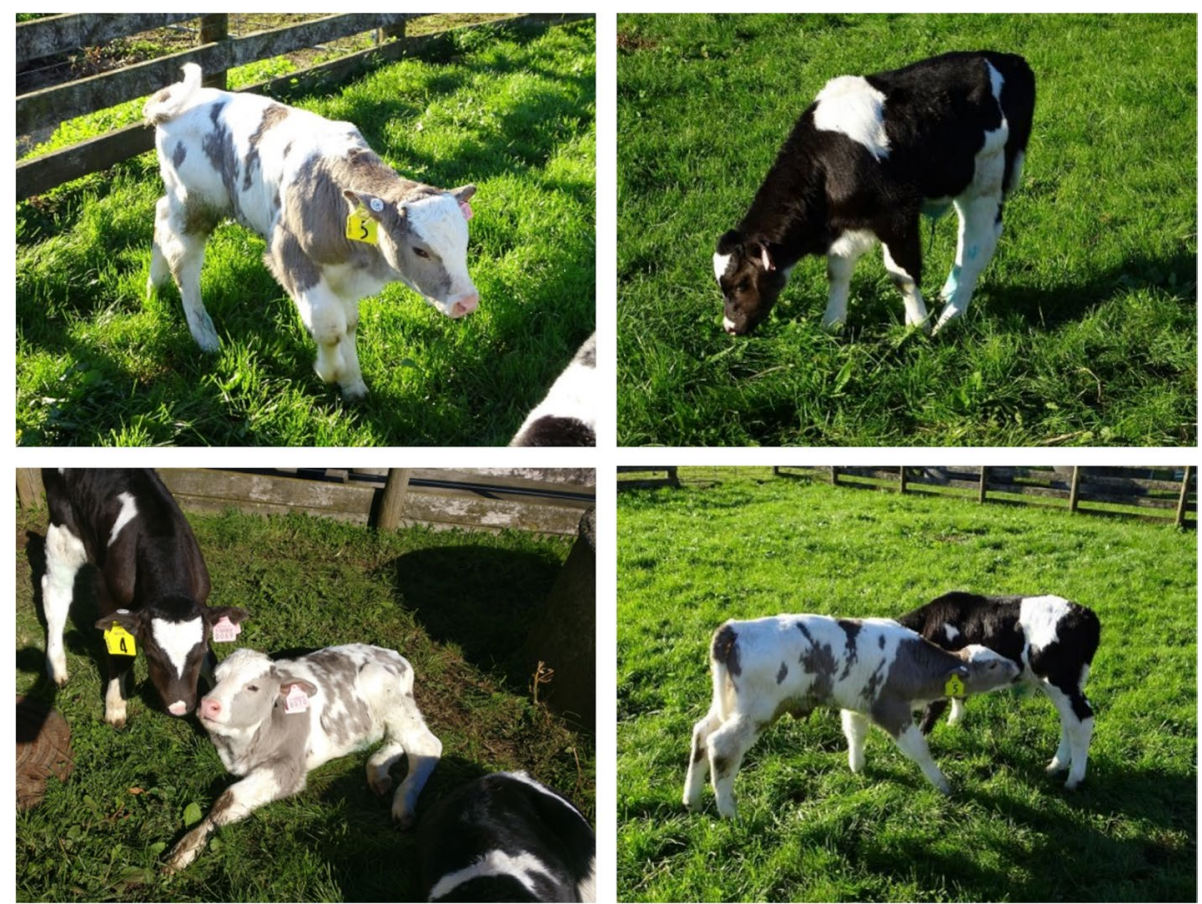

Fig. 3 Color dilution phenotype of genome edited calf. Shown are pictures of the PMEL mutant calf with non-edited control calves for direct comparison of coat colors and distribution of white and dark markings

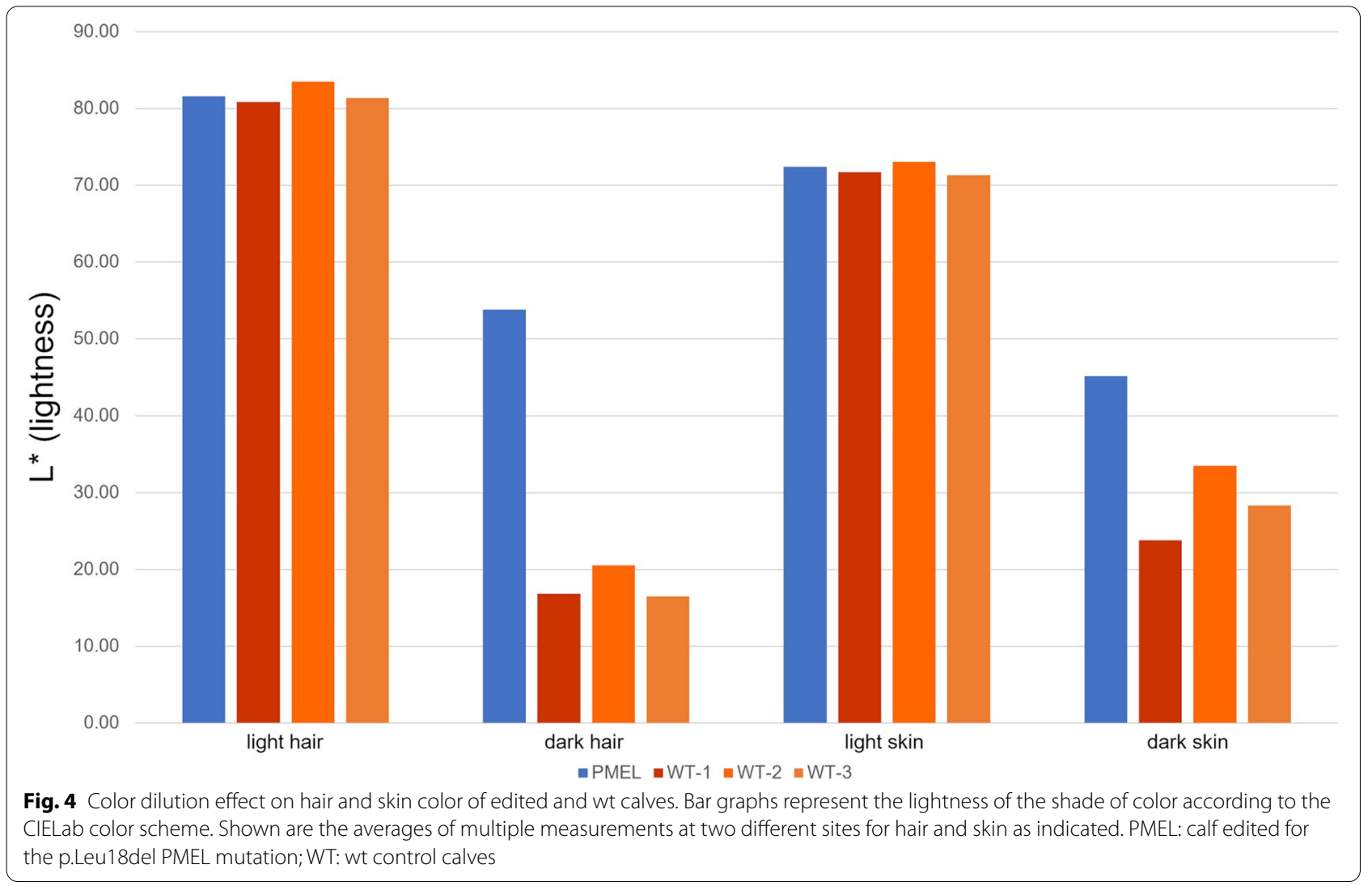


dark-haired coat markings. The $L^{*}$ value for skin beneath white-haired markings was the same for the PMEL mutant calf $\left(\mathrm{L}^{*}=72.4\right)$ and wt control calves $\left(\mathrm{L}^{*}=71.7\right.$, 73.0 and 71.3). However, similar to the difference observed for the darker hair, the shade of color for the skin under the darker coat markings showed a marked difference between the mutant calf $\left(\mathrm{L}^{*}=45.1\right)$ and the wt controls $\left(L^{*}=23.8,33.5\right.$ and $\left.28.3 ; P<0.0001\right)$.

\section{Impact of p.Leu18del on the processing of the PMEL signal peptide}

Considering the remarkable phenotypic impact of deleting a single leucine residue in the signal peptide of PMEL, we wondered about what impact p.Leu18del might have on the processing of the signal peptide, the $\mathrm{N}$-terminal end and overall length of the protein.

For wt bovine PMEL (UniProt entry Q06154), the database annotation specifies a 24 amino acid (aa) signal peptide. To assess the potential impact of the deleted leucine, we applied signal peptide predictions for wt and p.Leu18del PMEL sequences [23]. For both forms, presence of a signal peptide is predicted with a likelihood of 0.89 for wt and 0.85 for the mutated PMEL (Fig. 5). The cleavage site for the wt is predicted between aa 26 and aa 27 (TEG-PR) which differs from the database annotation. More importantly, the cleavage site predictions for wt are also different compared to the p.Leu18del PMEL variant. For the variant, the cleavage site was predicted between aa 19 and aa 20 (VLA-VG) which also provided supporting evidence that the signal peptide of the PMEL deletion variant is processed. Hence, the deletion of leucine 18 in the signal peptide is predicted to cause the generation of an N-terminal domain that differs in length (plus six aas) from wt PMEL. These results prompted us to examine the functionality of the signal peptide in another $\mathrm{N}$-terminal PMEL variant, p.Gly22Arg, that has been associated with coat color dilution in Charolais cattle [24]. Similar to the p.Leu18 del mutation, the signal peptide appears to be functional with a predicted likelihood of 0.83 and cleavage site between aa 23 and 24, extending the $\mathrm{N}$-terminal domain by two aas compared to wt PMEL (Fig. 5).

\section{Discussion}

The combination of the selected PMEL-specific gRNA/ Cas 9 editor and donor template specifying just the naturally occurring three bp deletion in the PMEL gene readily achieved precise, biallelic HDR outcomes with the ability to isolate cell clones with the intended precisely edited genotype. This suggests that the gRNA/Cas9 editor was sufficiently discriminatory for HDR- and wtallele to allow for precision editing without the need for introducing an additional mutation to eliminate the PAM motif. Although, a donor template with an additional PAM mutation might have further increased the achievable HDR efficiency. Overall, the ssODN repair template supported the accurate introduction of the intended precision edit without any potential changes at the strand invasion points.

Instead of using single cell sorting or antibiotic selection that might put cells under increased stress, we opted for manual picking of individual mitotic cells as a gentle method for the isolation of edited cell clones. A large proportion of transferred cells (75\%) successfully proliferated into expanded cell clones that were available for screening and subsequent production of edited calves. As expected from our low stringency PCR screen, rescreening of putative candidates with higher

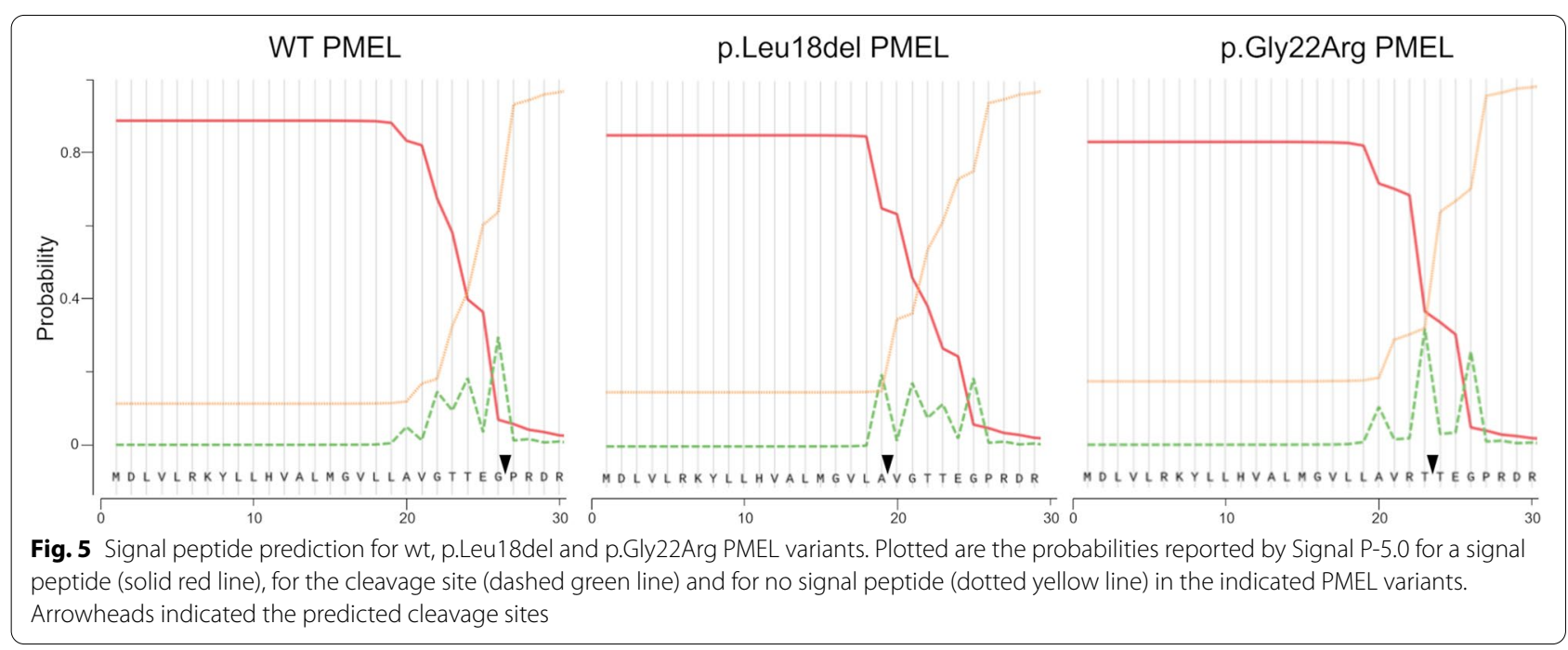


stringency identified several cell clones as false-positive. Even our stringent ddPCR assay using a hybridisation probe that was designed to selectively bind the correctly edited sequence showed some promiscuity. The assay also identified six cell clones, albeit at a lower (1000) than expected (2500) amplitude, that had a four bp instead of a three bp deletion at the target site (data not presented). Still, correctly edited cell clones could be readily identified by the assay and seven cell clones had genotypes with the precise, naturally occurring three bp deletion in the PMEL gene. Overall, the HDRediting efficiency proved to be sufficiently high (7/97; $7.3 \%$ ) to isolate several cell clones that were monoallelically and biallelically edited with precision by HDR.

The edited cells were suitable donor cells for SCNT and generated two cloned calves at term. The health of one of the PMEL calves was adversely affected by the effects from a hydrops pregnancy and was euthanized shortly after birth on welfare grounds. The second calf appeared healthy and thriving initially but was lost after 4 weeks due to a naval infection that went undetected. Both conditions are known complications associated with the generation of cloned cattle [25]. These developmental issues are assumed to be triggered by incorrect epigenetic reprogramming of the donor genome which renders the production of live cattle by cloning an inefficient process [26]. Although all three control calves remained healthy, the cloning success from edited and control donor cells was not significantly different $(P>0.1)$. Still, edited cells were put under additional stress from single cell clonal expansion and prolonged time in in vitro culture compared to control cells which might affect their clonability. Similar cloning-related inefficiencies in generating precisely edited cattle have been repeatedly reported in the literature [27-29]. This often means that only one or two live cloned founder calves are produced or might not result in a live calf at term [30]. The transfer of larger numbers of cloned embryos can provide greater certainty for the successful production of live cloned calves [31, 32]. However, access to bigger recipient herds, associated high costs and going against animal welfare efforts to reduce the number of animals for research are limitations of such a mitigation strategy. It is worth noting that the low efficiency hurdle only relates to the initial production of cloned edited 'founder' animals. Once they exist, large scale dissemination of the genetics will be possible by conventional reproductive technologies such as artificial insemination.

Low cloning efficiencies into viable edited calves of the cell-mediated editing approach could potentially be avoided altogether by directly editing in vitro produced zygotes that are not compromised in their developmental potential [33-35]. However, this approach has its own shortcomings. It lacks full control over the time and extent of editing and can generate complex mosaic genotypes [36,37], which to a certain extent can be addressed by screening embryos prior to transfer [38].

Both PMEL calves had the expected edited genotype of the precise biallelic three bp deletion in the PMEL gene that had been confirmed for the donor cell clone used for SCNT. Because the editor was delivered by a plasmid there is a potential for unintended vector integration. Although, for a circular, supercoiled plasmid integration events are rare [39], plasmids can integrate into random genomic loci [40] or in combination with site-specific editors, such as TALENS or gRNA/Cas9 nucleases, at the target site for double strand cleavage [41, 42]. The risk for such unintended integration of the molecular tools could be avoided by delivering editors as RNA molecules or as ribonucleoprotein complexes in the case of gRNA/Cas9, which can efficiently deliver editing activity into cells but are not substrates for possible integration into the genome [42]. Editors have also be shown to cause potential off-target mutations due to residual binding activity and thus, cleavage activity at sites that share sequence similarity with the target site [43]. However, when gRNAs are used that follow appropriate design rules, now provided by many online design tools, gRNA/Cas9 editors are unlikely to generate edited animals with significant off-target mutations [44]. Our testing of three predicted sites with the highest probability for an off-target event showed no evidence for sequence modifications at these non-target sites. To exclude that this targeted approach might miss potentially rare off-target events we have undertaken a comprehensive, unbiased analysis for any potential off-target mutations in a separate study [45]. Results confirmed that both calves were accurately edited for the intended three bp deletion and did not reveal any evidence for the presence of potential off-target mutations.

PMEL is a membrane protein that is expressed in pigmented cells where it is involved in the maturation of melanosomes, and pigment deposition and polymerisation in these organelles. It has a complex domain structure that has provided little insight into its function [46]. Mutations in PMEL were associated with black pigment dilution in several species but are found in different parts of the protein. While the cattle mutations in Charolais (p.Gly22Arg) as well as Highland and Galloway (p.Leu18del) are located within the signal peptide of PMEL $[18,24]$, in most other species, including mouse, chicken, dog and horses, naturally occurring functional mutations are clustered in the C-terminal transmembrane and cytoplasmic domains [12-16]. The more subtle mutations in cattle still produce a full-length protein. 
Assuming the predictions are correct, the mutant variants retain the functionality of the signal peptide. However, the cleavage site for the processing of the signal peptide is altered, generating a $\mathrm{N}$-terminus with six and three additional amino acids for the p.Leu18del and p.Gly22Arg mutations, respectively, that are part of the cleaved off signal peptide in the wt protein. These predictions still need to be experimentally verified by determining the $\mathrm{N}$-terminus of the variant forms of PMEL. If confirmed it would indicate that a seemingly minor change at the $\mathrm{N}$-terminus results in at least a partial loss of functionality, revealed by the observed hypopigmentation. This would be consistent with the important functional role attributed to the $\mathrm{N}$-terminal domain of PMEL [47] while the exact underlying molecular causes remain to be determined.

The two above mentioned mutations in the PMEL signal peptide were also reported to epistatically interact with $M C 1 R$. Animals that are heterozygous for the $P M E L$ mutation and have a copy of the dominant black MC1R allele $E^{\mathrm{D}}$ were associated with inherited hypotrichosis, also known as the "rat-tail" syndrome causing hair loss in pigmented areas $[48,49]$. By contrast, black animals with at least one $E^{\mathrm{D}}$ allele and homozygous for the $P M E L$ mutation appear to be unlikely to be affected by the hair loss [49]. The edited calves generated in this study were homozygous for $E^{\mathrm{D}}$ and the PMEL variant and, consistent with the above results, appeared to be unaffected by the "rat-tail" syndrome (Fig. S6), which might also be dependent on the interaction of a third so far unidentified locus [49]. In addition, evidence is emerging that PMEL is expressed in non-pigmented tissues suggesting additional potential functions beyond pigmentation [50].

Introgression of the homozygous p.Leu18del mutation into Holstein Friesian genetics resulted in a marked black pigment dilution effect. However, the dilution was not as strong compared to Highland cattle where, in homozygosity, the PMEL variant is associated with an almost white color, named silver-dun [18]. This partial rescue might be explained by interactions of $P M E L$ with additional genes that exist as different allelic variants in Highland and Holstein Friesian cattle. In Highland cattle, the p.Leu18 del mutation acts in a semi-dominant pattern. Whether this holds also true for Holstein Friesian cattle need still to be addressed by determining the coat color phenotype of heterozygous animals.

In addition, there was not only a general color dilution of black markings but a marked difference in the distribution and patterning of dark and white coat markings. The PMEL mutant calf had a larger total area of white markings and a characteristic white face compared to the control calves that were predominantly black with a black face and a white diamond shape on the forehead.
The differences go beyond what is normally observed for coat colour variations between cattle clones caused by some inherent epigenetic variability [51, 52]. Given that several, major effect QTL have recently been reported for white spotting in cattle of Holstein ancestry (modulated through the KIT, MITF, and PAX3 genes [22];), these findings might suggest potential epistatic interactions with these loci. Although the uniform genetic background of our study precludes a formal analysis in this regard, we genotyped tag SNPs and candidate causal mutations for these loci to provide context to the $P M E L$ derived observations and support potential future analyses to directly test this hypothesis in alternative genetic backgrounds. This analysis showed that our study animals carried a near full complement of 'white-increasing' alleles, suggesting introgression on other Holstein backgrounds might yield further (though incremental) gains in depigmentation. It is noteworthy that all four of these genes interact either directly or indirectly through shared pathways controlling melanoblast migration, differentiation, proliferation and/or survival [53, 54]. Epistatic interactions are therefore anticipated and might help further define the signalling relationships between these molecules and underlying melanocyte biology.

In summary, our study demonstrates the introgression of a precise PMEL mutation that naturally occurs in Highland cattle into both alleles of Holstein Friesian cattle. This mutation was associated with a coat color dilution phenotype in Highland cattle. When introgressed into Holstein Friesian cattle, it led to a coat color dilution effect, lightening the black coat markings to a silvery grey color. To our knowledge this is the first example that has functionally confirmed the causative status of a mutation for a coat color phenotype in cattle.

Considering the complexities of these genetics including the potential for yet unknown functions and epistatic interactions, the introduced PMEL mutation into Holstein-Friesian dairy genetics might have implications beyond the coat color change. However, the restriction to just a $3 \mathrm{bp}$ change can provide a valuable model to study the exact impacts not only on heat tolerance but also determine any potential effects it might have on reproductive performance, milk production characteristics, resilience to diseases associated with exposure to sunlight and general health and wellbeing. Moreover, such a model could also assist with elucidating the functional roles PMEL might play in non-pigmented tissues.

The rational of lightening the coat color was to alleviate heat stress and provide adaptation to warmer summer temperatures. Although we have demonstrated the precise editing of the p.Leu18del mutation for a dairy breed, the strategy could be readily applied to beef breeds such as Black Angus. Projected onto a global scale and 
considering the large number of low producing dairy cattle, even modest improvements of productivity through the use of color-diluted, high producing cattle that are adapted to warmer conditions would translate into substantial environmental benefits. However, a lighter coat color is only one aspect and introduction of additional sequence variants associated with improved thermotolerance will be required to further enhance the adaptation of grazing cattle. Despite the unfortunate loss of the edited calf to an infection, our study exemplified and provides support for genome editing as a new approach for the rapid adaptation of livestock to changing environmental conditions.

\section{Materials and methods Animal studies}

All animal experiments were performed in accordance with the relevant guidelines and regulations with approvals from New Zealand's Environmental Protection Authority (GMD100279) and the Ruakura Animal Ethics Committee (14236).

\section{Signal peptide prediction}

Signal peptide features of wt and deletion variant were evaluated using Signal P-5.0 [55].

\section{Genome editing}

PMEL-specific gRNAs were designed using the "CRISPOR" online tool [56]. The plasmid pX330 was modified by inserting high scoring gRNA sequences (Table S1) as previously described $[57,58]$. These expression plasmids were subsequently used to simultaneously deliver Cas9 nuclease and gRNA into male primary bovine fetal fibroblast (BEF2) cells. BEF2 cells were routinely cultured in Dulbecco's Modified Eagle's Medium (DMEM)/F12 supplemented with $10 \%$ fetal calf serum (Moregate Biotech). For editing, $2 \times 10^{5} \mathrm{BEF} 2$ cells were transfected with a gRNA/Cas9 plasmid $(0.5 \mu \mathrm{g})$ or cotransfected with a gRNA/Cas9 plasmid $(0.5 \mu \mathrm{g})$ plus HDR template $(0.4 \mu \mathrm{g})$ using a $10 \mu \mathrm{l}$ tip with program A3 (1500 V pulse voltage, $20 \mathrm{msec}$ pulse width, 1 pulse) according to the manufacturer's instruction of the Neon transfection system (Invitrogen). Following transfection, cells were reseeded and cultured for 2 days. After gently tapping the culture plate, dislodged mitotic cells were manually picked with a glass capillary and transferred into individual wells of a 96 well plate for the isolation of cell clones. After approximately 14days cell clones reached confluency and were successively transferred to larger multi-well plate formats for further expansion and cryopreservation.

\section{Genotype analysis}

Genomic DNA was extracted from cultured bovine cells and blood samples as previously described [59]. Sequences of all PCR primers used in this study are listed in Table S1.

The relevant region of the PMEL locus was PCR amplified with primer pair 1283/1284 using a Kapa 2G Fast Hotstart PCR kit (KapaBiosystems). Cycling conditions were $95^{\circ} \mathrm{C}, 3 \mathrm{~min} ; 95^{\circ} \mathrm{C}, 15 \mathrm{~s}, 60^{\circ} \mathrm{C}, 15 \mathrm{~s}$, and $72^{\circ} \mathrm{C}, 1 \mathrm{~s}$ for 35 cycles; and a final extension step of $72^{\circ} \mathrm{C}$ for $5 \mathrm{~min}$. For the mutation-specific PCR, the primer pair was $1249 / 1305$ and used the same amplification conditions as above. Similarly, the same conditions were used for the PCR-genotyping of the three 'white spotting' tag variants with primers representing the KIT (KIT_F/R), MITF (MITF_F/R), and PAX3 (PAX3_F/R) loci of interest.

For confirmation of the $M C 1 R$ alleles, genomic DNA was used to amplify the relevant regions containing the sequence variations, followed by sequencing as previously described [49]. Probing for potential gRNA/Cas9 plasmid integration was performed with the vector-specific primers 1041 and 795 amplifying a 489 bp fragment from the U6 promoter across the gRNA and into the $\mathrm{CMV}$ enhancer. Amplification of a 444bp fragment from the endogenous bovine alpha-lactalbumin gene ( $L A L B A)$ with primers 211 and 212 served as a control for the presence of amplifiable DNA. Cycle conditions were as detailed above.

The three genomic sites with the highest probability for off-target events (OFF1-3) as predicted by the CRISPOR design tool [56] for the bovine genome ARS-UCD1.2 were PCR amplified from genomic DNA as described above. Amplicons were sequenced and the sequence compared with the public bovine genome sequence to determine the presence of sequence changes at these sites in the edited genomes.

ddPCR assays were performed on a QX200 Droplet Digital PCR System (Bio-Rad) according to the method detailed in the Bio-Rad Droplet Digital ${ }^{\mathrm{TM}}$ PCR Applications Guide essentially as described before [38]. Briefly, ddPCR assays for non-homologous end joining (NHEJ) events were performed with approximately $100 \mathrm{ng}$ of genomic DNA as template, primer pair $1283 / 1284$, and a HEX-labelled drop-off (1287) and FAM-labelled reference probe (1286) located on the same amplicon. For determining template-mediated HDR editing, the dropoff probe was replaced by an HDR-probe (1285) and an unlabelled dark probe (1289). Following droplet generation, samples were PCR amplified using a Bio-Rad PCR machine as follows: $95^{\circ} \mathrm{C}$ for $10 \mathrm{~min}$, followed by 40 cycles of $94^{\circ} \mathrm{C} 30 \mathrm{~s}, 60^{\circ} \mathrm{C} 1 \mathrm{~min}$ (ramp rate $2^{\circ} \mathrm{C} / \mathrm{sec}$ ), then $98^{\circ} \mathrm{C}$ for $10 \mathrm{~min}$. Amplification results were acquired with a 
Bio-Rad Droplet Reader and analyzed using QuantaSoft ${ }^{\mathrm{TM}}$ Analysis Pro Software (Bio-Rad).

For sequence analyses, PCR reactions were separated on agarose gels and PCR fragments isolated using a Nucleo-Spin Gel and PCR Clean-up kit (MachereyNagel). Sanger sequencing of PMEL and KIT, MITF and $P A X 3$ fragments was provided by Massey Genome Service (Palmerston North, New Zealand) and the Auckland Genomics facility at the University of Auckland (Auckland, New Zealand), respectively. Tracking of Indels by DEcomposition (TIDE [60],) was used where required to dissect sequences of different gRNA/Cas9-generated mutations intertwined in the sequencing result of the amplified target region.

\section{SCNT}

SCNT embryos were reconstructed from abattoirderived, enucleated oocytes and individual, serum starved-donor cells using a zona-free cloning procedure as previously described [59]. After 7 days in vitro culture single embryos were non-surgically transferred to synchronized recipient cows for development to term.

\section{Hair and skin color measurements}

The lightness of color was measured using a Hunter Miniscan XE colorimeter (HunterLab, Hunter Associates Laboratories Inc., Reston, USA) according to the CIELAB color system [61]. The lightness of color is measured as an $\mathrm{L}^{*}$ value that is defined by the position on the black (0) to white (100) axis of the visible spectrum. For each of the three control calves, the $L^{*}$ values were determined for the hair of two different white and two dark markings, with one area located on the right and the other on the left flank of each animal. To determine the corresponding values for skin, the hair from a small area in these markings was shaved and $L^{*}$ values were measured for two light and two dark markings. For the PMEL mutant calf, the coat/pelt was removed following the death of the animal and used for the color measurements. Again, two white and two dark markings on opposing flanks were selected and $\mathrm{L}^{*}$ determined with three individual measurements each.

The clipped hair were further used to analyse differences in hair length.

\section{Statistical analysis}

Statistical significance levels of observed differences were determined by the two-tailed Fisher exact test for independence in $2 \times 2$ tables (cloning efficiency of CC14 vs wt) and two-tailed student's t-test ( $L^{*}$ measurements).

\section{Supplementary Information}

The online version contains supplementary material available at https://doi. org/10.1186/s12864-021-08175-z.

Additional file 1: Table S1. PCR primer, probe, gRNA and repair template sequences used to characterise the PMEL locus and white spotting genes. Table S2. Genotype for major-effect QTL associated with white spotting. Fig. S1. Target site sequence of the genome edited calves. Fig. S2. The edited claves are homozygous for the MC1R ED allele. Fig. S3. Absence of a plasmid-specific fragment in genomic DNA from edited calves. Fig. S4. Original gel photo used to generate Fig. S3. Fig. S5. Absence of mutations at the top three predicted off-target sites. Fig. S6. Absence of characteristic hair phenotypes associated with rat tail syndrome.

\section{Acknowledgements}

We thank Stephanie Delaney and Ruakura farm staff for dedicated animal husbandry, Fanli Meng and Pavla Turner for assistance with SCNT and Suzanne Rowe for critical reading of the manuscript. This work was funded by AgResearch and the Ministry of Business, Innovation and Employment.

\section{Authors' contributions}

Conceptualization: GL, MDL. Formal analysis: GL. Funding acquisition: GL, MDL, DNW. Investigation: GL, SAC, BB, JW, SL, SJ, DNW. Methodology: BB, SL, SJ, DNW. Project administration: GL. Supervision: GL. Validation: GL, SAC, BB, JW, SL, SJ, DNW. Visualization: GL. Writing - original draft: GL. Writing - review \& editing: GL, BB, SL, SJ, MDL, DNW. All authors read and approved the submission of the manuscript.

\section{Funding}

This work was funded through the Ministry of Business, Innovation and Employment (https://www.mbie.govt.nz/) Endeavour Funds CONT-62639-ENDRP-AGR and CONT-57639-ENDRP-LIC. The funders had no role in study design, data collection and analysis, decision to publish, or preparation of the manuscript.

\section{Availability of data and materials}

All data generated or analysed during this study are included in this published article and its supplementary information files.

\section{Declarations}

\section{Ethics approval and consent to participate}

All animal experiments were performed in accordance with the relevant guidelines and regulations with approvals from New Zealand's Environmental Protection Authority (GMD100279) and the Ruakura Animal Ethics Committee (14236).

The study was carried out in compliance with the ARRIVE guidelines.

\section{Consent for publication}

Not applicable.

\section{Competing interests}

G.L., S-A.C., B.B., J.W., S.L. and D.N.W. are employees of AgResearch and declare that they have no conflict of interest or financial conflicts to disclose. Likewise, S. J declares to not have competing interests. M.D.L. is an employee of Livestock Improvement Corporation, a commercial provider of bovine germplasm.

\section{Author details}

${ }^{1}$ AgResearch, Ruakura Research Centre, Hamilton 3240, New Zealand. ${ }^{2}$ School of Medical Sciences, University of Auckland, Auckland, New Zealand. ${ }^{3}$ Maurice Wilkins Centre for Molecular Biodiscovery, Auckland, New Zealand. ${ }^{4}$ Massey University Manawatu, Palmerston North, New Zealand. ${ }^{5}$ Livestock Improvement Corporation, Newstead, Hamilton, New Zealand. 
Received: 7 July 2021 Accepted: 22 October 2021

Published online: 26 November 2021

\section{References}

1. Chambers LE, Griffiths GM. The changing nature of temperature extremes in Australia and New Zealand. Aust Meteorol Mag. 2008;57(1):13-35.

2. Easterling DR, Meehl GA, Parmesan C, Changnon SA, Karl TR, Mearns LO. Climate extremes: observations, modeling, and impacts. Science. 2000;289(5487):2068-74.

3. Fischer EM, Knutti R. Anthropogenic contribution to global occurrence of heavy-precipitation and high-temperature extremes. Nat Clim Chang. 2015;5(6):560-4

4. Seneviratne SI, Donat MG, Mueller B, Alexander LV. No pause in the increase of hot temperature extremes. Nat Clim Chang. 2014;4(3):161-3.

5. Kadzere CT, Murphy MR, Silanikove N, Maltz E. Heat stress in lactating dairy cows: a review. Livest Prod Sci. 2002;77(1):59-91.

6. Nardone A, Ronchi B, Lacetera N, Ranieri MS, Bernabucci U. Effects of climate changes on animal production and sustainability of livestock systems. Livest Sci. 2010;130(1-3):57-69.

7. Wheelock JB, Rhoads RP, VanBaale MJ, Sanders SR, Baumgard LH. Effects of heat stress on energetic metabolism in lactating Holstein cows. J Dairy Sci. 2010;93(2):644-55.

8. Bryant JR, López-Villalobos N, Pryce JE, Holmes CW, Johnson DL. Quantifying the effect of thermal environment on production traits in three breeds of dairy cattle in New Zealand. N Z J Agric Res. 2007;50(3):327-38.

9. Stewart RE. Absorption of solar radiation by the hair of cattle. Agric Eng. 1953:34:235-8.

10. Hansen PJ. Effects of coat colour on physiological responses to solar radiation in Holsteins. Vet Rec. 1990;127(13):333-4.

11. King VL, Denise SK, Armstrong DV, Torabi M, Wiersma F. Effects of a hot climate on the performance of first lactation Holstein cows grouped by coat color. J Dairy Sci. 1988;71(4):1093-6.

12. Brunberg E, Andersson L, Cothran G, Sandberg K, Mikko S, Lindgren G. A missense mutation in PMEL17 is associated with the silver coat color in the horse. BMC Genet. 2006;7:46.

13. Clark LA, Wahl JM, Rees CA, Murphy KE. Retrotransposon insertion in SILV is responsible for merle patterning of the domestic dog. Proc Natl Acad Sci U S A. 2006;103(5):1376-81.

14. Kerje S, Sharma P, Gunnarsson U, Kim H, Bagchi S, Fredriksson R, et al. The dominant white, dun and smoky color variants in chicken are associated with insertion/deletion polymorphisms in the PMEL17 gene. Genetics. 2004; 168(3):1507-18.

15. Kwon BS, Halaban R, Ponnazhagan S, Kim K, Chintamaneni C, Bennett $D$, et al. Mouse silver mutation is caused by a single base insertion in the putative cytoplasmic domain of Pmel 17. Nucleic Acids Res. 1995;23(1):154-8.

16. Martinez-Esparza M, Jimenez-Cervantes C, Bennett DC, Lozano JA, Solano F, Garcia-Borron JC. The mouse silver locus encodes a single transcript truncated by the silver mutation. Mamm Genome. 1999;10(12):1168-71.

17. Schonthaler HB, Lampert JM, von Lintig J, Schwarz H, Geisler R, Neuhauss SC. A mutation in the silver gene leads to defects in melanosome biogenesis and alterations in the visual system in the zebrafish mutant fading vision. Dev Biol. 2005;284(2):421-36.

18. Schmutz SM, Dreger DL. Interaction of MC1R and PMEL alleles on solid coat colors in Highland cattle. Anim Genet. 2013;44(1):9-13.

19. Davis SR, Spelman RJ, Littlejohn MD. BREEDING AND GENETICS SYMPOSIUM:Breeding heat tolerant dairy cattle: the case for introgression of the "slick" prolactin receptor variant into dairy breeds. J Anim Sci. 2017;95(4):1788-800

20. Laible G, Wei J, Wagner S. Improving livestock for agriculture - technological progress from random transgenesis to precision genome editing heralds a new era. Biotechnol J. 2015;10(1):109-20.

21. McLean Z, Oback B, Laible G. Embryo-mediated genome editing for accelerated genetic improvement of livestock. Front Agr Sci Eng. 2020;7:148-60.

22. Jivanji S, Worth G, Lopdell TJ, Yeates A, Couldrey C, Reynolds E, et al. Genome-wide association analysis reveals QTL and candidate mutations involved in white spotting in cattle. Genet Sel Evol. 2019;51(1):62.
23. Almagro Armenteros JJ, Tsirigos KD, Sonderby CK, Petersen TN, Winther O, Brunak S, et al. SignalP 5.0 improves signal peptide predictions using deep neural networks. Nat Biotechnol. 2019:37(4):420-3.

24. Kuhn C, Weikard R. An investigation into the genetic background of coat colour dilution in a Charolais x German Holstein F2 resource population. Anim Genet. 2007;38(2):109-13.

25. Wells DN. Animal cloning: problems and prospects. Rev Sci Tech. 2005;24(1):251-64.

26. Oback B. Climbing mount efficiency--small steps, not giant leaps towards higher cloning success in farm animals. Reprod Domest Anim. 2008:43(Suppl 2):407-16.

27. Carlson DF, Lancto CA, Zang B, Kim ES, Walton M, Oldeschulte D, et al. Production of hornless dairy cattle from genome-edited cell lines. Nat Biotechnol. 2016;34(5):479-81.

28. Perota A, Lagutina I, Duchi R, Zanfrini E, Lazzari G, Judor JP, et al. Generation of cattle knockout for galactose-alpha1,3-galactose and N-glycolylneuraminic acid antigens. Xenotransplantation. 2019;26(5):e12524.

29. Su X, Wang S, Su G, Zheng Z, Zhang J, Ma Y, et al. Production of microhomologous-mediated site-specific integrated LacS gene cow using TALENs. Theriogenology. 2018:119:282-8.

30. Carvalho BP, Cunha ATM, Silva BDM, Sousa RV, Leme LO, Dode MAN, et al. Production of transgenic cattle by somatic cell nuclear transfer (SCNT) with the human granulocyte colony-stimulation factor (hG-CSF). J Anim Sci Technol. 2019;61(2):61-8.

31. Gao Y, Wu H, Wang Y, Liu X, Chen L, Li Q, et al. Single Cas9 nickase induced generation of NRAMP1 knockin cattle with reduced off-target effects. Genome Biol. 2017;18(1):13.

32. Wu H, Wang Y, Zhang Y, Yang M, Lv J, Liu J, et al. TALE nickase-mediated SP110 knockin endows cattle with increased resistance to tuberculosis. Proc Natl Acad Sci U S A. 2015;112(13):E1530-9.

33. Wei J, Wagner S, Lu D, Maclean P, Carlson DF, Fahrenkrug SC, et al. Efficient introgression of allelic variants by embryo-mediated editing of the bovine genome. Sci Rep. 2015;5:11735.

34. Park KE, Frey JF, Waters J, Simpson SG, Coutu C, Plummer S, et al. Onestep homology mediated CRISPR-Cas editing in zygotes for generating genome edited cattle. CRISPR J. 2020;3(6):523-34.

35. Proudfoot C, Carlson DF, Huddart R, Long CR, Pryor JH, King TJ, et al. Genome edited sheep and cattle. Transgenic Res. 2015;24(1):147-53.

36. Hennig SL, Owen JR, Lin JC, Young AE, Ross PJ, Van Eenennaam AL, et al. Evaluation of mutation rates, mosaicism and off target mutations when injecting Cas 9 mRNA or protein for genome editing of bovine embryos. Sci Rep. 2020;10(1):22309.

37. Owen JR, Hennig SL, McNabb BR, Mansour TA, Smith JM, Lin JC, et al. One-step generation of a targeted knock-in calf using the CRISPR-Cas9 system in bovine zygotes. BMC Genomics. 2021;22(1):118.

38. Wei J, Wagner S, Maclean P, Brophy B, Cole S, Smolenski G, et al. Cattle with a precise, zygote-mediated deletion safely eliminate the major milk allergen beta-lactoglobulin. Sci Rep. 2018;8(1):7661.

39. Wurtele $\mathrm{H}$, Little KC, Chartrand P. Illegitimate DNA integration in mammalian cells. Gene Ther. 2003;10(21):1791-9.

40. Graham C, Cole S, Laible G. Site-specific modification of the bovine genome using Cre recombinase-mediated gene targeting. Biotechnol J. 2009:4(1):108-18.

41. Young AE, Mansour TA, McNabb BR, Owen JR, Trott JF, Brown CT, et al. Genomic and phenotypic analyses of six offspring of a genome-edited hornless bull. Nat Biotechnol. 2020;38(2):225-32.

42. Kim S, Kim D, Cho SW, Kim J, Kim JS. Highly efficient RNA-guided genome editing in human cells via delivery of purified Cas9 ribonucleoproteins. Genome Res. 2014;24(6):1012-9.

43. Fu Y, Foden JA, Khayter C, Maeder ML, Reyon D, Joung JK, et al. Highfrequency off-target mutagenesis induced by CRISPR-Cas nucleases in human cells. Nat Biotechnol. 2013;31(9):822-6.

44. Akcakaya P, Bobbin ML, Guo JA, Malagon-Lopez J, Clement K, Garcia SP, et al. In vivo CRISPR editing with no detectable genome-wide off-target mutations. Nature. 2018;561(7723):416-9.

45. Jivanji S, Harland C, Cole S, Brophy B, Garrick D, Snell RG, et al. The genomes of precision edited cloned calves show no evidence for off-target events or increased de novo mutagenesis. BMC Genomics. 2021;22:457.

46. Theos AC, Truschel ST, Raposo G, Marks MS. The silver locus product Pmel17/gp100/Silv/ME20: controversial in name and in function. Pigment Cell Res. 2005;18(5):322-36. 
47. Mitchell SM, Graham M, Liu X, Leonhardt RM. Identification of critical amino acid residues in the regulatory $\mathrm{N}$-terminal domain of PMEL. Sci Rep. 2021;11(1):7730.

48. Hauser M, Wolf-Hofstetter S, Acklin-Menzi F, Studer E, Rediger D, Seefried F, et al. Grey, curly and short-haired Swiss Holstein cattle show genetic traces of the Simmental breed. Schweiz Arch Tierheilkd. 2020;162(9):551-9.

49. Knaust J, Hadlich F, Weikard R, Kuehn C. Epistatic interactions between at least three loci determine the "rat-tail" phenotype in cattle. Genet Sel Evol. 2016;48:26.

50. Knaust J, Weikard R, Albrecht E, Brunner RM, Gunther J, Kuhn C. Indication of Premelanosome Protein (PMEL) Expression Outside of Pigmented Bovine Skin Suggests Functions Beyond Eumelanogenesis. Genes (Basel). 2020;11(7):788.

51. Brophy B, Smolenski G, Wheeler T, Wells D, L'Huillier P, Laible G. Cloned transgenic cattle produce milk with higher levels of beta-casein and kappa-casein. Nat Biotechnol. 2003;21(2):157-62.

52. Wells DN, Misica PM, Tervit HR. Production of cloned calves following nuclear transfer with cultured adult mural granulosa cells. Biol Reprod. 1999:60(4):996-1005

53. D'Mello SA, Finlay GJ, Baguley BC, Askarian-Amiri ME. Signaling Pathways in Melanogenesis. Int J Mol Sci. 2016;17(7):1144.

54. Du J, Miller AJ, Widlund HR, Horstmann MA, Ramaswamy S, Fisher DE. MLANA/MART1 and SILV/PMEL17/GP100 are transcriptionally regulated by MITF in melanocytes and melanoma. Am J Pathol. 2003;163(1):333-43.
55. CBS. SignalP-5.0. 2019

56. Haeussler M, Schonig K, Eckert H, Eschstruth A, Mianne J, Renaud JB, et al. Evaluation of off-target and on-target scoring algorithms and integration into the guide RNA selection tool CRISPOR. Genome Biol. 2016;17(1):148

57. Cong L, Ran FA, Cox D, Lin S, Barretto R, Habib N, et al. Multiplex genome engineering using CRISPR/Cas systems. Science. 2013;339(6121):819-23.

58. ZhangLab. Target sequence cloning protocol 2013 [Available from: https://media.addgene.org/cms/filer_public/e6/5a/e65a9ef8-c8ac-4f8898da-3b7d7960394c/zhang-lab-general-cloning-protocol.pdf.

59. Jabed A, Wagner S, McCracken J, Wells DN, Laible G. Targeted microRNA expression in dairy cattle directs production of beta-lactoglobulin-free, high-casein milk. Proc Natl Acad Sci U S A. 2012;109(42):16811-6.

60. Brinkman EK, Chen T, Amendola M, van Steensel B. Easy quantitative assessment of genome editing by sequence trace decomposition. Nucleic Acids Res. 2014;42(22):e168.

61. Hunter RS, Harold RW. The measurement of appearance. 2nd ed. New York: Wiley; 1987. p. 411

\section{Publisher's Note}

Springer Nature remains neutral with regard to jurisdictional claims in published maps and institutional affiliations.
Ready to submit your research? Choose BMC and benefit from:

- fast, convenient online submission

- thorough peer review by experienced researchers in your field

- rapid publication on acceptance

- support for research data, including large and complex data types

- gold Open Access which fosters wider collaboration and increased citations

- maximum visibility for your research: over $100 \mathrm{M}$ website views per year

At BMC, research is always in progress.

Learn more biomedcentral.com/submissions 